\title{
Outcomes of endoscopic ultrasound and endoscopic resection of gastrointestinal subepithelial lesions: a single-center retrospective cohort study
}

\author{
Ariosto H. Hernandez-Lara a, Ana Garcia Garcia de Paredesa, Louis M. Wong Kee Songa, \\ Daniel J. Rowan ${ }^{b}$, Rondell P. Graham ${ }^{b}$, Michael J. Levya, Ferga C. Gleesona, Amrit K. Kamboja, \\ Kristin C. Marac, Barham K. Abu-Dayyeh ${ }^{a}$, Vinay Chandrasekharaa ${ }^{a}$ Prasad G. Iyera, Andrew C. Storma, \\ Elizabeth Rajan ${ }^{\text {a }}$
}

Mayo Clinic, Rochester, Minnesota, USA

Abstract

\begin{abstract}
Background Endoscopic resection (ER) is an emerging therapeutic alternative for subepithelial gastrointestinal lesions (SELs). We aimed to determine whether size, layer of origin, and histology based on endoscopic ultrasound (EUS) and EUS-guided sampling (EUS-GS) influenced the outcomes and selection of patients for ER.
\end{abstract}

Methods We performed a retrospective review of patients who underwent EUS, EUS-GS and resection of SELs from 2012-2019. Two pathologists reviewed the histology and layer of origin of all resected specimens, serving as the criterion for EUS accuracy.

Results Seventy-three patients were included, of whom 59 (81\%) were gastric SELs. Per EUS, median lesion size was $21 \mathrm{~mm}$ (interquartile range 15-32), and 63 (86\%) originated from the 4th layer. The overall accuracy of EUS and EUS-GS in predicting the layer of origin and histology was $88 \%$ (95\% confidence interval [CI] 77-94\%) and 96\% (95\%CI 87-98\%), respectively. Based on EUS, 18 (25\%) patients were referred for ER, 5 (7\%) to laparoscopic-endoscopic cooperative surgery, and $50(68 \%)$ to surgery. Size $>20 \mathrm{~mm}$ was associated with the type of resection approach $(\mathrm{P}=0.005)$, while layer of origin and histology were not $(\mathrm{P}=0.06$ and $\mathrm{P}=0.09$, respectively). When SELs were inaccurately classified $(n=4)$ there were no adverse events or revision of the resection approach.

Conclusions EUS plays an important role in the outcome of resection approach for SELs, with size significantly influencing the selection for ER. In patients undergoing ER, no revised resections were needed when EUS was inaccurate.

Keywords Endoscopic ultrasound, endoscopic resection, subepithelial lesion

Ann Gastroenterol 2021; 35 (1): 1-5

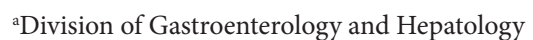

(Ariosto H. Hernandez-Lara, Ana Garcia Garcia de

Paredes, Louis M. Wong Kee Song, Michael J. Levy, Ferga C. Gleeson,

Amrit K. Kamboj, Barham K. Abu-Dayyeh, Vinay Chandrasekhara,

Prasad G. Iyer, Andrew C. Storm, Elizabeth Rajan); 'Department

of Anatomic Pathology (Daniel J. Rowan, Rondell P. Graham);

'Department of Biomedical Statistics and Informatics (Kristin C. Mara),

Mayo Clinic, Rochester, Minnesota, USA

Conflict of Interest: None

Correspondence to: Elizabeth Rajan MD, FASGE, Professor of Medicine, Division of Gastroenterology and Hepatology, Mayo Clinic, 200 First Street SW, Rochester, Minnesota, 55905, USA,

e-mail: rajan.elizabeth16@mayo.edu

Funding: This study was supported by a grant from the Division of Gastroenterology and Hepatology, Mayo Clinic, Rochester, MN (grant number UL1TR002377)

Received 30 November 2020; accepted 3 February 2021; published online 2 April 2021

DOI: https://doi.org/10.20524/aog.2021.0621

\section{Introduction}

Gastrointestinal tract subepithelial lesions (SELs) are often incidentally found during endoscopy or on radiologic examination. The true prevalence of SELs is unknown. A retrospective study reported a prevalence of $0.36 \%$ for gastric SELs after routine endoscopy [1]. Histologic types of SELs include benign entities, such as lipoma and pancreatic rest, and potentially malignant lesions, such as gastrointestinal stromal tumors. The reported diagnostic accuracy of endoscopic ultrasound (EUS) for SELs is $77 \%$, which increases to $91 \%$ when EUS-guided sampling is performed [2].

SELs have traditionally been treated by surgical resection [3]; however, endoscopic resection is fast emerging as a therapeutic alternative. EUS is commonly undertaken to evaluate and diagnose SELs by identifying the layer of origin, echo features, size of the lesion and tissue sampling, in order 
to guide management from surveillance to resection [4]. There remains a paucity of data on whether performing EUS and EUS-guided sampling actually impacts the outcome or the selection of the resection approach. Well-defined indications for endoscopic resection are yet to be established, and not all SELs are amenable to endoscopic resection. Therefore, the aims of this study were to assess whether lesion size, layer of origin, and histology of SELs, based on EUS and EUS-guided sampling, influence the outcomes and selection of patients for endoscopic resection.

\section{Patients and methods}

\section{Study design and population}

A retrospective review was performed of patients who underwent EUS, EUS-guided sampling, and endoscopic or surgical resection of gastrointestinal SELs from January 2012 to December 2019. Only lesions in sites that can be accessed and evaluated by EUS (esophagus, stomach, duodenum or rectum) were included. Data on patient demographics, EUS findings, tissue acquisition, pathology, resection technique and adverse events were obtained from electronic medical records and a procedural database (Provation Medical Inc., Minneapolis, $\mathrm{MN})$. The study was approved by the Institutional Review Board (IRB\# 19-006474).

\section{EUS and EUS-guided sampling}

All EUS procedures were performed by experienced endosonographers. Procedures were performed using Olympus (Olympus Medical Systems, Center Valley, PA) or Pentax (Pentax, Montvale, NJ) echoendoscope systems. Needles used for EUS-guided tissue acquisition were $19 \mathrm{G}, 22 \mathrm{G}$ or $25 \mathrm{G}$ (EchoTip Ultra and Quick-Core, Cook Medical, Bloomington, IN; SharkCore, Medtronic, Minneapolis, MN) at the discretion of the endoscopist.

\section{Resection}

After EUS and EUS-guided sampling evaluation, patients were referred for endoscopic resection, laparoscopicendoscopic cooperative surgery (LECS) or surgical resection. Two pathologists independently reviewed the histologic type and layer of origin of all resected specimens that served as the criterion standard to determine the accuracy of EUS.

\section{Statistical analysis}

Descriptive statistics are reported as frequencies and percentages for categorical variables, and as mean \pm standard deviation or median and interquartile range (IQR), as appropriate, for continuous variables. For the inferential analysis, $t$-tests were used to compare continuous data and chi-square tests were used to compare categorical data. Lesion size on EUS and cross-sectional imaging was correlated by calculating the Spearman correlation coefficient (rho, $\rho$ ). All analyses were 2-tailed and statistical significance was set at $\mathrm{P}<0.05$. All statistical tests were performed using the software package JMP Pro 14, SAS Institute Inc. Cary, North Carolina, United States.

\section{Results}

\section{Study population and EUS}

A total of 73 patients met the study inclusion criteria (mean age $63 \pm 14$ years, $51 \%$ male.) EUS was indicated after abnormal computed tomography scan (CT) in $34(47 \%)$ patients and for suspected SEL found during endoscopy in 39 (53\%). Indications for CT and/or endoscopy that resulted in EUS included evaluation and surveillance of tumors unrelated to SEL: e.g., renal cancer $(n=13,17.8 \%)$, dyspepsia $(n=10,13.7 \%)$, abdominal pain $(n=10,13.7 \%)$, iron deficiency anemia and overt gastrointestinal bleeding $(n=10,13.7 \%)$, gastroesophageal reflux disease $(n=8,11 \%)$, and miscellaneous reasons, such as bloating ( $n=22,30.1 \%)$.

The majority of SELs were located in the stomach (81\%). The median size of SELs was $21 \mathrm{~mm}$ (IQR 15-32), while 44 $(60 \%)$ lesions were $>20 \mathrm{~mm}$. Sixty of the 73 patients $(82 \%)$ had cross-sectional imaging $(\mathrm{CT}=58$, magnetic resonance imaging=2) with a median lesion size of $26 \mathrm{~mm}$ (IQR 17.5-42). There was a positive strong correlation in size between EUS and cross-sectional imaging, which was statistically significant $(\rho=0.88, P<0.001)$. Per EUS, 63 (86\%) SELs originated from the 4th layer (muscularis propria) of the gastrointestinal tract and 71 (97\%) were hypoechoic. Fine needle aspiration (FNA), fine needle biopsy (FNB), and FNA + FNB were performed in $29 \%, 48 \%$, and $23 \%$ of cases, respectively. The overall diagnostic yield of EUS-guided sampling was $92 \%$, with no significant difference in yield between FNA and FNB techniques $(\mathrm{P}=0.86)$. Lymphadenopathy was reported in 5 (7\%) patients. There were no adverse events related to EUS and EUS-guided sampling. Detailed information is displayed in Table 1 .

\section{Resection}

Based on EUS and EUS-guided sampling, 50 (68\%) patients were referred for surgical resection, 18 (25\%) for endoscopic resection and $5(7 \%)$ for LECS. There were no significant differences in age $(\mathrm{P}=0.97)$, body mass index $(\mathrm{P}=0.32)$ or $\mathrm{SEL}$ location $(\mathrm{P}=0.26)$ between the resection approaches.

Methods for endoscopic resection included endoscopic full thickness resection (EFTR) in nine (50\%) patients, submucosal tunneling endoscopic resection (STER) in 4 (22\%), endoscopic 
Table 1 Patient baseline characteristics and EUS features of gastrointestinal subepithelial lesions

\begin{tabular}{lc}
\hline Patients' characteristics & $\mathrm{n}=73$ \\
\hline Male sex & $37(51 \%)$ \\
\hline Age (years; mean \pm SD) & $63 \pm 14$ \\
EUS & \\
SEL location & \\
Stomach & $59(81 \%)$ \\
Duodenum & $8(11 \%)$ \\
Esophagus & $4(5 \%)$ \\
Rectum & $2(3 \%)$ \\
SEL size (mm; median [IQR]) & $21(15-32)$ \\
Layer of origin & \\
$4^{\text {th }}$ (Muscularis propria) & $63(86 \%)$ \\
$3^{\text {rd }}$ (Submucosa) & $6(8 \%)$ \\
$2^{\text {nd }}$ (Deep mucosa) & $4(6 \%)$ \\
Regional lymphadenopathy & \\
No & $68(93 \%)$ \\
Yes & $5(7 \%)$ \\
Echogenicity & \\
Hypoechoic & $71(98 \%)$ \\
Hyperechoic & $1(1 \%)$ \\
Anechoic & $1(1 \%)$ \\
Tissue acquisition & \\
FNB & \\
FNA & $35(48 \%)$ \\
FNA +FNB & $21(29 \%)$ \\
Needle gauge & $17(23 \%)$ \\
22 G & \\
19 G & \\
25 G & $(96 \%)$ \\
\hline SD, standard deviation; IQR, interquartile range; EUS, endoscopic ultrasound; \\
SEL, subepithelial lesion; FNB, fine needle biopsy; FNA, fine needle aspiration
\end{tabular}

submucosal dissection (ESD) in 4 (22\%), and endoscopic mucosal resection (EMR) in $1(6 \%)$. Endoscopic resection success rate was $94 \%$, with adverse events reported in $2(3 \%)$ patients. Both occurred during peroral lesion extraction: an esophageal tear and an esophageal perforation managed with endoscopic clips and surgery, respectively.

\section{Role of EUS in selection of resection approach}

Gastrointestinal stromal tumor was the most common definitive diagnosis $(46,62 \%)$. The overall accuracy of EUS and EUS-guided sampling in predicting the layer of origin and histologic type were $88 \%$ (95\% confidence interval [CI] 77-94\%) and 96\% (95\%CI 87-98\%), respectively. The highest and lowest agreements between EUS and layer of origin of resected specimens were for gastrointestinal stromal tumors and neuroendocrine tumors, respectively, while pancreatic heterotopia had the lowest agreement between EUS-guided sampling and the pathology of the resected specimen (Table 2). There were no malignant lesions seen in our cohort of patients.
Table 2 EUS and EUS-guided sampling agreement with resected specimen

\begin{tabular}{lccc}
\hline Pathology & $\mathrm{N}=73(\%)$ & $\begin{array}{c}\text { Layer of origin } \\
\text { agreement }\end{array}$ & $\begin{array}{c}\text { Histologic type } \\
\text { agreement }\end{array}$ \\
\hline GIST & $45(62 \%)$ & $92 \%$ & $100 \%$ \\
Leiomyoma & $11(15 \%)$ & $86 \%$ & $100 \%$ \\
NET & $5(7 \%)$ & $40 \%$ & $80 \%$ \\
$\begin{array}{l}\text { Pancreatic } \\
\text { heterotopia }\end{array}$ & $4(5 \%)$ & $100 \%$ & $0 \%$ \\
Lipoma & $2(3 \%)$ & $100 \%$ & $100 \%$ \\
$\begin{array}{l}\text { Neural } \\
\text { neoplasm }\end{array}$ & $2(3 \%)$ & $50 \%$ & $100 \%$ \\
Others & $4(5 \%)$ & $100 \%$ & $100 \%$ \\
\hline
\end{tabular}

EUS, endoscopic ultrasound; GIST, gastrointestinal stromal tumor; NET, neuroendocrine tumor

In univariate analysis, lesion size $>20 \mathrm{~mm}$ measured by EUS significantly influenced the selection of resection approach $(\mathrm{P}=0.005)$. Layer of origin and histologic type did not influence resection approach $(\mathrm{P}=0.06$ and $\mathrm{P}=0.09$, respectively).

\section{EUS-related outcomes of endoscopic resection}

In patients who had endoscopic resection of SELs, the accuracy of EUS and EUS-guided sampling in predicting the layer of origin and histologic type was $71 \%$ (95\%CI $45-$ $88 \%)$ and $100 \%$ (95\%CI $81-100 \%)$, respectively. In patients in whom SELs were inaccurately classified based on layer of origin $(n=4)$ there were no adverse events reported or need for a revised resection approach, such an example is depicted by Fig. 1A-F.

\section{Discussion}

Gastrointestinal SELs are traditionally resected with surgery. Endoscopic resection is fast emerging as an alternative approach; however, the indications for endoscopic treatment of SELs are not well-established. Our study evaluated the role of EUS in selecting the resection approach for SELs, and found that lesion size assessed by EUS significantly influenced the decision on mode of resection.

EUS evaluation and EUS-guided sampling of SELs provide information on lesion size, layer of origin and histologic type $[5,6]$. EUS is frequently recommended as the modality of choice to characterize SELs. Studies have shown that the overall accuracy of EUS alone in differentiating SELs ranges from $43-77 \%$. In a study by Akahoshi et al, the diagnostic yield was related to the size of SEL: $71 \%, 86 \%$ and $100 \%$ for lesions measuring $<20 \mathrm{~mm}, 20-40 \mathrm{~mm}$ and $>40 \mathrm{~mm}$, respectively [7]. The use of endosonographic criteria such as lesion size (>20 mm), irregular margins, inhomogeneous pattern or presence of lymph nodes $(>10 \mathrm{~mm})$ can improve differentiation 

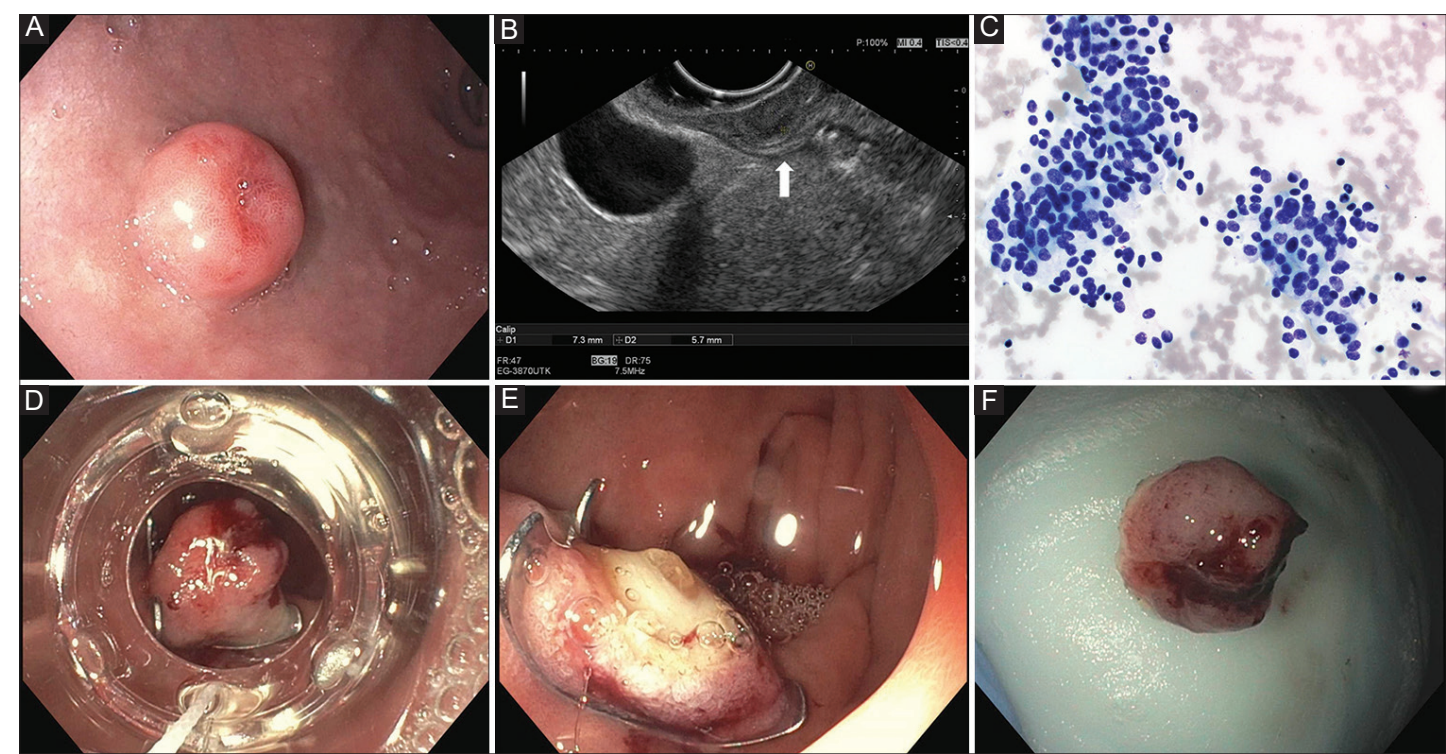

Figure 1 (A) Duodenal bulb subepithelial lesion. (B) EUS demonstrates the subepithelial lesion originated from the second layer (arrow). (C) Cells from FNA show pale cytoplasm, round nucleus with salt and pepper chromatin, suggesting a well-differentiated neuroendocrine tumor (Pap stain. 400× original magnification). (D) Over-the-scope clip-assisted endoscopic full thickness resection with the neuroendocrine subepithelial lesion above the deployed clip. (E) Post-resection site. (F) En bloc resected specimen, which on pathology showed clear margins and the layer of origin of the neuroendocrine tumor to be from the submucosa (layer 3)

EUS, endoscopic ultrasound; FNA, fine needle aspiration

for malignant lesions with a specificity of $80 \%$, but with a relatively low sensitivity of $64 \%[8,9]$. EUS-guided sampling improved the diagnostic yield to $>80 \%[5,10-16]$. In our cohort there were no differences in diagnostic accuracy between FNA and FNB; however, a recent meta-analysis favored FNB over FNA in patients with SELs [17].

Information about the accuracy, outcomes and role of EUS in the selection of patients undergoing endoscopic vs. surgical resection of SELs is scarce. In our study cohort, the overall accuracy of EUS in predicting the layer of origin was $88 \%$, while the accuracy of EUS-guided sampling in identifying histologic type was $96 \%$, similar to previous studies [18]. In a subgroup analysis of patients who underwent endoscopic resection, the accuracy of EUS and EUS-guided sampling in predicting the layer of origin and histologic type was $71 \%$ and $100 \%$, respectively. This highlights the challenges in identifying the layer of origin, particularly with ill-defined lesions arising from the third layer of the gut wall [11]. Based on EUS findings, 32\% of SELs were considered suitable candidates for endoscopic resection and LECS, while $68 \%$ were referred for surgical resection. In the univariate analysis, only size $>20 \mathrm{~mm}$ was significantly associated with the resection approach. The low number of endoscopic resections in our cohort $(\mathrm{n}=18)$ precluded a multivariate analysis to identify independent factors associated with endoscopic resection. Layer of origin and histologic type did not significantly influence the resection approach in our cohort of patients; however, they did allow the differentiation of benign from malignant entities and provide a roadmap for the choice of endoscopic technique (e.g., EMR for superficial lesions and EFTR for deeper lesions). Layer of origin was inaccurately assessed by EUS in 4 patients who underwent endoscopic resection, with no adverse clinical outcomes or need for a revised resection approach. At present, there are no guidelines or standardized criterion based on size for referral of gastrointestinal SELs for surgery vs. endoscopic resection. Even though some authors suggest endoscopic removal of lesions $<3-4 \mathrm{~cm}$ [19], in most studies the mean size of lesions treated by endoscopic resection is close to $20 \mathrm{~mm}$ [20]. This is in keeping with our study, in which most lesions $>20 \mathrm{~mm}$ were referred for surgery.

Endoscopic resection techniques for SELs include EFTR, STER and ESD, with reported success rates ranging from $90-100 \%$ [19,21-23]. In our study, the most common endoscopic technique was EFTR and the overall success rate of endoscopic resection was $94 \%$. Adverse events were reported in $3 \%$ of our patients undergoing endoscopic resection. Complication rates related to endoscopic resection are heterogeneous among different series in the literature, with reports of bleeding ranging from $0-24 \%$ and perforation from $0-5 \%[24,25]$.

Although EUS is commonly used to diagnose SELs in clinical practice, its role in determining the optimal type of resection is unclear. This study highlights the merits and benefits of EUS when selecting gastrointestinal SELs for endoscopic resection. The study is limited by the small sample size, single-center experience, inherent limitations of a retrospective study and potential selection bias based on physician referral.

Based on our study, EUS should be considered prior to selecting a resection approach for gastrointestinal SELs, with size significantly influencing the selection for endoscopic resection. In our cohort of patients undergoing endoscopic resection, no revisions of resection or adverse events occurred when EUS was inaccurate. 


\section{Summary Box}

\section{What is already known:}

- Endoscopic resection is emerging as a therapeutic alternative to surgery for gastrointestinal subepithelial lesions (SELs)

- Indications for endoscopic resection of SELs are not yet established

- There are limited data on the role and outcomes of endoscopic ultrasound (EUS) in the selection of patients for endoscopic vs. surgical resection

\section{What the new findings are:}

- Lesion size assessed by EUS influences the selection mode for resection of SELs

- The accuracy of EUS in predicting the layer of origin was $88 \%$; in patients undergoing endoscopic resection, no revision of resection or adverse events occurred when EUS was inaccurate

- EUS should be considered prior to selecting a resection approach for gastrointestinal SELs

\section{References}

1. Hedenbro JL, Ekelund M, Wetterberg P. Endoscopic diagnosis of submucosal gastric lesions. The results after routine endoscopy. Surg Endosc 1991;5:20-23.

2. Karaca C, Turner BG, Cizginer S, Forcione D, Brugge W. Accuracy of EUS in the evaluation of small gastric subepithelial lesions. Gastrointest Endosc 2010;71:722-727.

3. Ponsaing LG, Hansen MB. Therapeutic procedures for submucosal tumors in the gastrointestinal tract. World $J$ Gastroenterol 2007;13:3316-3322.

4. Shim CS, Jung IS. Endoscopic removal of submucosal tumors: preprocedure diagnosis, technical options, and results. Endoscopy 2005;37:646-654.

5. Ando N, Goto H, Niwa Y, et al. The diagnosis of GI stromal tumors with EUS-guided fine needle aspiration with immunohistochemical analysis. Gastrointest Endosc 2002;55:37-43.

6. Chak A. EUS in submucosal tumors. Gastrointest Endosc 2002;56:S43-S48.

7. Akahoshi K, Sumida Y, Matsui N, et al. Preoperative diagnosis of gastrointestinal stromal tumor by endoscopic ultrasound-guided fine needle aspiration. World J Gastroenterol 2007;13:2077-2082.

8. Rösch T, Kapfer B, Will U, et al; German EUS Club. Endoscopic ultrasonography. Accuracy of endoscopic ultrasonography in upper gastrointestinal submucosal lesions: a prospective multicenter study. Scand J Gastroenterol 2002;37:856-862.

9. Eckardt AJ, Jenssen C. Current endoscopic ultrasound-guided approach to incidental subepithelial lesions: optimal or optional? Ann Gastroenterol 2015;28:160-172.

10. Polkowski M, Butruk E. Submucosal lesions. Gastrointest Endosc Clin N Am 2005; 15:33-54.

11. Hwang JH, Saunders MD, Rulyak SJ, Shaw S, Nietsch H, Kimmey MB. A prospective study comparing endoscopy and EUS in the evaluation of GI subepithelial masses. Gastrointest Endosc 2005;62:202-208.

12. Suzuki T, Arai M, Matsumura T, et al. Factors associated with inadequate tissue yield in EUS-FNA for gastric SMT. ISRN Gastroenterol 2011;2011:619128.

13. Hoda KM, Rodriguez SA, Faigel DO. EUS-guided sampling of suspected GI stromal tumors. Gastrointest Endosc 2009;69:1218-1223.

14. Polkowski M, Gerke W, Jarosz D, et al. Diagnostic yield and safety of endoscopic ultrasound-guided trucut [corrected] biopsy in patients with gastric submucosal tumors: a prospective study. Endoscopy 2009;41:329-334.

15. Fernández-Esparrach G, Sendino O, Solé M, et al. Endoscopic ultrasound-guided fine-needle aspiration and trucut biopsy in the diagnosis of gastric stromal tumors: a randomized crossover study. Endoscopy 2010;42:292-299.

16. Han JP, Lee TH, Hong SJ, et al. EUS-guided FNA and FNB after on-site cytological evaluation in gastric subepithelial tumors. J Dig Dis 2016;17:582-587.

17. Facciorusso A, Sunny SP, Del Prete V, Antonino M, Muscatiello N. Comparison between fine-needle biopsy and fine-needle aspiration for EUS-guided sampling of subepithelial lesions: a meta-analysis. Gastrointest Endosc 2020;91:14-22.

18. Zhang XC, Li QL, Yu YF, et al. Diagnostic efficacy of endoscopic ultrasound-guided needle sampling for upper gastrointestinal subepithelial lesions: a meta-analysis. Surg Endosc 2016;30:2431-2441.

19. Zhang X, Modayil R, Criscitelli T, Stavropoulos SN. Endoscopic resection for subepithelial lesions-pure endoscopic full-thickness resection and submucosal tunneling endoscopic resection. Transl Gastroenterol Hepatol 2019;4:39.

20. Kim SY, Kim KO. Endoscopic treatment of subepithelial tumors. Clin Endosc 2018;51:19-27.

21. Al-Bawardy B, Rajan E, Wong Kee Song LM. Over-the-scope clip-assisted endoscopic full-thickness resection of epithelial and subepithelial GI lesions. Gastrointest Endosc 2017;85:1087-1092.

22. Rajan E, Wong Kee Song LM. Endoscopic full thickness resection. Gastroenterology 2018;154:1925-1937.

23. Aslanian HR, Sethi A, Bhutani MS, et al; ASGE Technology Committee. ASGE guideline for endoscopic full-thickness resection and submucosal tunnel endoscopic resection. VideoGIE 2019;4:343-350.

24. Cantor MJ, Davila RE, Faigel DO. Yield of tissue sampling for subepithelial lesions evaluated by EUS: a comparison between forceps biopsies and endoscopic submucosal resection. Gastrointest Endosc 2006;64:29-34.

25. Hunt GC, Smith PP, Faigel DO. Yield of tissue sampling for submucosal lesions evaluated by EUS. Gastrointest Endosc 2003;57:68-72. 